\title{
ANH
}

\section{CASOS DE MESOTELIOMA MALIGNO EM 20 ANOS DE UM REGISTRO HOSPITALAR DE CÂNCER}

\author{
Tatiana Lage Ferreira Halfeld ${ }^{1}$, Larissa Marcondes ${ }^{2}$, Luciana Puchalski Kalinke ${ }^{3}$,
} Dario Consonni ${ }^{4}$, Carolina Mensi' ${ }^{5}$ Leila Maria Mansano Sarquis ${ }^{6}$.

\section{RESUMO}

Objetivo: Analisar a qualidade dos dados dos casos de mesotelioma maligno notificados no Registro Hospitalar de Câncer em um hospital oncológico de Curitiba-PR.

Método: Estudo retrospectivo, transversal, realizado em um Centro de Alta Complexidade em Oncologia na cidade de Curitiba, Paraná, no período de janeiro a dezembro de 2017. A amostra foi composta por 142 registros de pacientes adultos com câncer de topografia (Classificação Internacional das Doenças para Oncologia (CID-O) $3^{a}$ Revisão) C38 (câncer do coração, mediastino e pleura) e C48 (câncer do retroperitônio e peritônio) durante o período de janeiro de 1995 a dezembro de 2015. Para as análises estatísticas, foi utilizado o software Stata $15 \AA$.

Resultados: Foram identificados 16 casos de mesotelioma maligno (11 pleurais e 5 retroperitoneais), diagnosticados a partir de exames anatomopatológico e de imuno-histoquímica. Destes casos, três foram notificados com topografias inadequadas. Divergências foram encontradas na comparação entre os diagnósticos registrados em prontuários e os registrados em cinco Declarações de Óbito. A sensibilidade do Registro Hospitalar de Câncer de um hospital oncológico referência em Curitiba ao notificar mesotelioma maligno foi de $81 \%$ e a especificidade ao notificar câncer de pleura foi de $97 \%$.

Conclusão: Houve subnotificação nos casos de mesotelioma maligno (13 em vez de 16) e uma sobrestimativa foi observada nas notificações de cânceres de pleura (4 em vez de 0 ), decorrente da codificação equívoca da descrição da doença por parte do registro. Diagnóstico precoce, integralidade no acompanhamento clínico, capacitação dos profissionais envolvidos na geração e interpretação dos dados são pilares fundamentais para a correta definição da epidemiologia do mesotelioma maligno.

Descritores: Mesotelioma; Asbestos; Saúde pública; Epidemiologia; Sistemas de informação.

1. Tatiana Lage Ferreira Halfeld - Enfermeira. Mestre em Enfermagem. Universidade Federal do Paraná. Programa de Pós-Graduação em Enfermagem. Curitiba, PR, Brasil. tatianahalfeld@gmail.com - ORCID - https://orcid.org/0000-0001-9572-3989

2. Larissa Marcondes - Enfermeira. Mestre em Enfermagem. Universidade Federal do Paraná. Programa de Pós-Graduação em Enfermagem. Curitiba, PR, Brasil. marcondes.lari@gmail.com - ORCID - https://orcid.org/0000-0002-8745-6486

3. Luciana Puchalski Kalinke - Enfermeira. Doutora em Ciências da Saúde. Universidade Federal do Paraná. Programa de Pós-Graduação em Enfermagem. Curitiba, PR, Brasil. lucianakalinke@yahoo.com.br - ORCID - https://orcid.org/0000-0003-4868-8193

4. Dario Consonni - Médico. Doutor em Epidemiologia. Fondazione IRCCS Ca' Granda Ospedale Maggiore Policlinico, Unidade de Epidemiologia, Milão, Lombardia, Itália. dario.consonni@unimi.it - ORCID - https://orcid.org/0000-0002-8935-3843.

5. Carolina Mensi - Biológa. Doutora em Epidemiologia. Fondazione IRCCS Ca' Granda Ospedale Maggiore Policlinico, Unidade de Epidemiologia, Milão, Lombardia, Itália. carolina.mensi@unimi.it - ORCID - https://orcid.org/0000-0002-9075-3684

6. Leila Maria Mansano Sarquis - Enfermeira. Doutora em Enfermagem. Universidade Federal do Paraná. Programa de Pós-Graduação em Enfermagem. Curitiba, PR, Brasil. Immsarquis@gmail.com - ORCID - https://orcid.org/0000-0002-0542-5062

\section{Autor Correspondente}

Tatiana Lage Ferreira Halfeld

Endereço: Av. Prefeito Lothário Meissner, 632 - Campus Botânico -

Bloco Didático II - 30 andar - Jd. Botânico - Curitiba, PR. - 80210-170.

E-mail: tatianahalfeld@gmail.com Telefone: (41) 99871-5441
Data de submissão: 08/06/2019

Data de aceite: $19 / 08 / 2019$

Seção a que o manuscrito se destina: Artigo oiginal.

Como citar esse artigo

HALFELD, T. L. F.; et al. Casos de mesotelioma maligno em 20 anos de um registro hospitalar de câncer. Advances in Nursing and Health, v. 1, p. 85-97, Londrina, 2019. 


\section{INTRODUÇÃO}

O mesotelioma maligno (MM) é definido como um câncer agressivo e incurável, de rápida evolução para o óbito, e é proveniente das células mesoteliais e submesoteliais que compõem a pleura ( $>90 \%$ dos casos), peritônio, pericárdio e túnica vaginal dos testículos[1]. Além disso, o prognóstico desse tipo de câncer pode envolver metástase em linfonodos adjacentes, pulmões, fígado, pâncreas, rins, glândulas suprarrenais e medula óssea[2].

Segundo a International Agency for Research on Cancer (IARC), desde 1960 o principal fator ambiental reconhecido no desenvolvimento do MM está relacionado à inalação e deposição de fibras de amianto ou asbestos em tecido respiratório. Essas fibras são derivadas de um grupo de rochas metamórficas naturalmente encontradas na natureza e amplamente utilizadas como matéria prima industrial[3].

Na maioria dos casos de MM nos homens (e em menor parte nas mulheres) a exposição ao amianto está relacionada ao ambiente de trabalho, o que permite defini-lo como uma doença ocupacional[4-5]. No entanto, também existe a possibilidade de contaminação através da exposição ambiental[6] ou ainda paraocupacional por meio do contato com fibras impregnadas em roupas de trabalhadores que tiveram contato prévio com amianto, ou durante a manipulação de objetos compostos por tais fibras, como luvas e mantas térmicas[7].

Devido ao reconhecimento de seu potencial carcinogênico na década de 70, o amianto está banido, em todas as suas formas (serpentinas - crisotila e anfibólios - crocidolita, amosita, tremolita, antofilita, actinolita), em mais de 60 países. 0 Brasil iniciou o seu uso em 1940, mantendo um crescimento vertiginoso cujo ápice ocorreu entre 1985 e 1991, quando foram banidos os anfibólios). Recentemente, o Brasil ainda era considerado um dos cinco maiores produtores, consumidores e exportadores do tipo crisotila, também chamado de amianto branco[8].

Pesquisas que levam em consideração o histórico particular das regiões produtoras e consumidoras de amianto têm constatado que a incidência e a prevalência de mesotelioma vêm aumentando progressivamente. Para o Brasil, até 2030 estão estimadas cerca de 1.911 mortes por MM, sendo que o pico será entre 2021 e 2026. Já para os casos de neoplasia maligna de pleura (tumores 
primários sem confirmação histológica), são estimadas 2.406 mortes, com pico em período similar ao das mortes por MM. Juntas, as duas doenças totalizam uma estimativa de 4.301 mortes. Esses índices são mais tardios em relação às estimativas de mortes nos demais países industrializados em cerca de 15 a 20 anos, o que é compatível com a cronologia de consumo do Brasil[4].

Diante dos estudos confirmando a nocividade do amianto, bem como as inúmeras iniciativas de diversas entidades e do Ministério Público do Trabalho em defesa da extinção do seu uso, o Supremo Tribunal Federal (STF) declarou, no dia 29 de novembro de 2017, a proibição da extração, industrialização e comercialização em território nacional de qualquer produto que contenha amianto crisotila em sua composição. Além disso, essa declaração também comunicou que o Congresso e os Estados não poderiam aprovar leis para autorizar o uso da fibra[9]. No entanto, o banimento do amianto soluciona apenas parcialmente o problema gerado pela fibra, tendo em vista que são necessários cerca de 40 anos ou mais para a manifestação do MM[10]. Por esta razão, o acompanhamento de trabalhadores, seus familiares, e pessoas expostas ambientalmente ao amian- to é fundamental para o conhecimento e o controle dos riscos para a população[11-12].

Uma das estratégias adotadas no Brasil para identificar e acompanhar os casos de câncer em território nacional é o Registro Hospitalar de Câncer (RHC) Esse registro é uma fonte sistemática de informações, que de forma estratégica é instalado em hospitais gerais ou especializados em oncologia. Essa iniciativa tem como objetivo coletar dados relativos ao diagnóstico, tratamento e evolução dos casos confirmados de câncer atendidos nessas instituições, sejam elas públicas, privadas, de ensino ou filantrópicas. Juntamente com os Registros de Câncer de Base Populacional (RCBPs) e o Sistema de Informação de Mortalidade (SIM), esse sistema forma o eixo estruturante da política de atenção ao câncer no país. Em conjunto, esses sistemas são considerados pelo Instituto Nacional de Câncer (INCA) como "ferramentas poderosas para a vigilância epidemiológica do câncer no país"[13].

O RHC possui uma função clínica, ao oferecer informações valiosas à administração do hospital que devem ser usadas para beneficiar diretamente seus usuários. A partir de seus indicadores, a qualidade da assistência oferecida pelo hospital pode ser 
verificada, inclusive os resultados alcançados durante o tratamento do câncer. Além disso, o RHC assume também o importante papel de monitorar e avaliar a assistência prestada a esses pacientes, fornecendo dados estatísticos que precisam ser confiáveis, uma vez que auxiliam a equipe de saúde a nortear suas condutas.

Este estudo busca descrever a qualidade dos diagnósticos dos casos de MM e neoplasias malignas de pleura e peritônio atendidos em um hospital de referência em oncologia de Curitiba.

\section{MÉTODO}

A análise dos dados foi elaborada através de um estudo retrospectivo e transversal, realizado no RHC de um Centro de Alta Complexidade em Oncologia (CACON) na cidade de Curitiba-PR, localizada na região sul do Brasil, durante o período de janeiro a dezembro de 2017.

Os critérios de inclusão dos prontuários foram: pacientes $\geq 18$ anos no momento da admissão na instituição, Classificação Internacional das Doenças para Oncologia (CID-0) $3^{a}$ Revisão com topografia C38 e C48 ou com morfologia M905_/3 (tumor maligno), para tumores malignos primitivos de coração (C38.0), mediastino (C38.1, C38.2, C38.3), pleura (C38.4), retroperitônio (C48.0), peritônio (C48.1, C48.2), códigos morfológicos específicos para o mesotelioma maligno $(9050 / 3,9051 / 3,9052 / 3$ e 9053/3) e notificação durante o período de 1995 a 2015. A temporalidade das buscas foi estabelecida de acordo com a disponibilidade para a consulta das informações registradas no RHC pesquisado.

Inicialmente, foi realizada a coleta de dados no integrador RHC da instituição (iRHC), cuja codificação é CID-O3. Nesta etapa, foram identificados 54 prontuários com códigos do Grupo C38 e 88 prontuários com códigos do Grupo C48, totalizando 142 prontuários elegíveis. Após essa triagem, dados provenientes de prontuários físicos e eletrônicos (quando disponíveis) foram extraídos com o auxílio de um instrumento de coleta de dados.

Para a realização do estudo, os dados foram tabulados no programa Microsoft Excel $® 2010$ e os resultados obtidos foram expressos tanto em análise quanto em frequência simples ( $n$ ) e absoluta (\%) a intervalos de confiança (IC) de $95 \%$. 


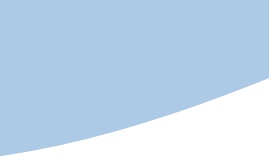

Para verificar a sensibilidade (Se) e especificidade (Sp) do RHC, foi elaborado um quadro com base na morfologia, no diagnóstico encontrado no RHC e nos laudos anatomopatológico (AP) e/ou de imunohistoquímica (IHQ) (padrão ouro) presentes nos prontuários analisados. Essas informações foram avaliadas por serem relativas aos registros da evolução médica dos pacientes e aos laudos dos exames que fundamentaram os diagnósticos. Posteriormente, realizou-se as codificações dos diagnósticos, conforme o preconizado pela CID-O3. Após esta etapa, todos os códigos encontrados (utilizados pelo RHC e os obtidos após a coleta dos dados) foram convertidos em CID-10 (Classificação Internacional de Doenças (CID) $10^{a}$ Revisão). Dessa forma, casos com morfologia de mesotelioma maligno (CID-O-3: 90503, 90513, 90523, 90533) foram reclassificados como C45 (CID-10). Os resultados foram expressos em frequências simples ( $n$ ) e absolutas (\%) e intervalos de confiança (IC) de 95\%. As análises estatísticas foram realizadas através do software Stata $15 \AA$.

Este estudo foi aprovado pelo Comitê de Ética em Pesquisa da instituição cenário da pesquisa, sob o número 2514, respaldado pela Resolução 466/2012.
RESULTADO

A partir dos dados do RHC, foram identificados 54 casos de câncer com topografia C38 (Neoplasia maligna do coração, mediastino e pleura) e 88 casos de câncer com topografia C48 (Neoplasia maligna do retroperitônio e do peritônio), totalizando 142 casos.

A avaliação dos diagnósticos encontrados em prontuários demonstrou que dos casos registrados com códigos do grupo C38, 38 eram relativos a lesões mediastinais, sendo que: dois casos em Mediastino Anterior (C38.1), 35 casos em Mediastino Sem Outra Especificação (SOE, C38.3) e um caso em Coração (C38.0). Entre os 15 casos identificados pelo código C38.4 (Pleura, SOE), 11 eram referentes a pacientes diagnosticados com Mesotelioma Maligno de Pleura; os demais se referiam ao uso do código para a notificação de casos de Linfoma Não Hodgkin, Carcinoma Escamo Celular (CEC) e Adenocarcinoma.

Dos 88 casos de câncer retroperitoneal e peritoneal, 74 ocorreram em Retroperitônio (C48.0), 10 ocorreram em Peritônio, SOE (C48.2), quatro em partes específicas do Peritônio (C48.1) e um com lesão sobreposta 
de Retroperitônio e Peritônio (C48.8). Apenas cinco casos de MM foram identificados.

Constatou-se que a Se do RHC para MM (C45) foi de $81 \%$ (95\% CI: 54-96\%), uma vez que três dos 16 casos da doença foram notificados com topografias inapropriadas (C49.3, C48.0 e C34.9) (tabela 1). Destaca-se que a topografia inapropriada invalida a notificação da doença, mesmo que o código morfológico esteja correto, uma vez que a conversão final para o CID-10 equivalente é inviável. O exame dos prontuários não mostrou nenhum caso de C38.4.

Quanto à $\mathrm{Sp}$ do registro ao notificar câncer da pleura (tabela 1), apresenta-se com 97\% (95\% CI: 92-99\%), sendo que em quatro casos esse câncer foi notificado erroneamente como C38.4 (falsos positivos, um câncer de pulmão, um câncer de mediastino e dois cânceres com metástase em pleura).

Tabela 1 - Sensibilidade e especificidades do Registro Hospitalar de Câncer para a notificação de casos de mesotelioma maligno. Códigos CID-10. Curitiba, PR, Brasil, 2017.

\begin{tabular}{|c|c|c|c|c|}
\hline \multirow[b]{2}{*}{ RHC } & \multicolumn{4}{|c|}{ Prontuário } \\
\hline & $\begin{array}{c}\text { C45 } \\
\text { Mesotelioma }\end{array}$ & $\begin{array}{c}\text { C38.4 } \\
\text { Pleura }\end{array}$ & Outro câncer & Total \\
\hline C45 - Mesotelioma & $13(81 \%) *$ & 0 & $0(0 \%)$ & 13 \\
\hline C38.4 - Pleura & $0(0 \%)$ & 0 & 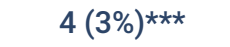 & 4 \\
\hline Outro câncer & $3(19 \%)^{\star \star}$ & 0 & 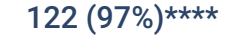 & 125 \\
\hline Total & $16(100 \%)$ & 0 & $126(100 \%)$ & 142 \\
\hline \multicolumn{5}{|c|}{ 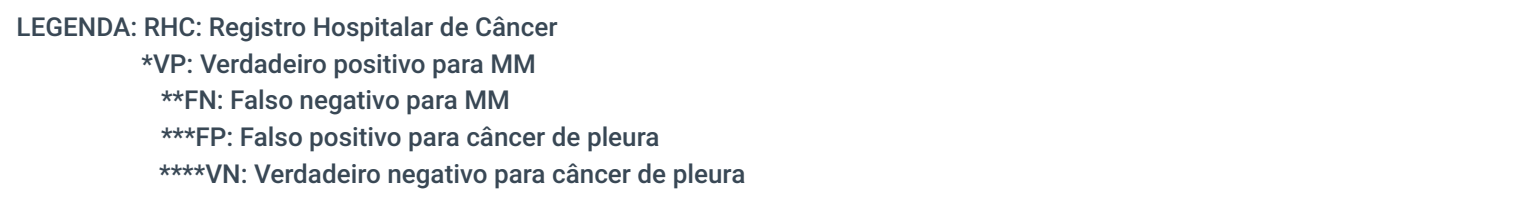 } \\
\hline \multicolumn{2}{|c|}{ A observação da cronologia das admis- } & \multicolumn{3}{|c|}{ relativas ao óbito em qualquer circunstância } \\
\hline \multicolumn{2}{|c|}{ sões e óbitos mostrou que houve menos de } & \multicolumn{3}{|c|}{ em prontuários de três pacientes (tabela 2 ). A } \\
\hline \multicolumn{2}{|c|}{ uma admissão de MM/ano. Foram encontra- } & \multicolumn{3}{|c|}{ sobrevida mediana foi de 10,2 meses (nos 9} \\
\hline \multicolumn{2}{|c|}{ dos registros de comunicação por parte de } & \multicolumn{3}{|c|}{ MM pleurais: 6,8 meses; nos 4 MM peritone- } \\
\hline \multicolumn{2}{|c|}{ familiares de dois óbitos ocorridos fora da ins- } & \multicolumn{3}{|c|}{ ais: 11,4 meses). Apenas para um dos casos } \\
\hline tituição e outros 10 oc & urante a in- & \multicolumn{3}{|c|}{ (técnico em tubulação) foi reportada a expo- } \\
\hline ternação. Não foram enc & hformações & \multicolumn{3}{|c|}{ sição ao amianto no prontuário. } \\
\hline
\end{tabular}


Tabela 2 - Casos de mesotelioma maligno registrados no Registro Hospitalar de Câncer e avaliados no prontuário (códigos CID-03) no período de 1995 a 2015. Curitiba, PR, Brasil, 2017.

\begin{tabular}{|c|c|c|c|c|c|c|c|c|}
\hline Sexo & $\begin{array}{c}\text { Idade ao } \\
\text { diagnóstico }\end{array}$ & $\begin{array}{c}\text { Ano de } \\
\text { diagnóstico }\end{array}$ & P TOPO & P MORFO & RHC TOPO & RHC MORFO & $\begin{array}{l}\text { Sobrevida } \\
\text { (meses) }\end{array}$ & Profissão \\
\hline$F$ & 53 & 2002 & C48.2 & $9052 / 3$ & C48.1 & $9050 / 3$ & 17,9 & $\begin{array}{l}\text { Trabalhadores do } \\
\text { comércio e assemelhados }\end{array}$ \\
\hline M & 58 & 2010 & C38.4 & $9053 / 3$ & C38.4 & $9050 / 3$ & NR & NR \\
\hline M & 56 & 2006 & C48.2 & $9050 / 3$ & C48.2 & $9050 / 3$ & 12,6 & $\begin{array}{l}\text { Cozinheiros e } \\
\text { trabalhadores } \\
\text { assemelhados }\end{array}$ \\
\hline M & 75 & 2003 & C38.4 & $9052 / 3$ & C49.3 & 90503 & 5,6 & $\begin{array}{l}\text { Trabalhadores do } \\
\text { comércio e assemelhados }\end{array}$ \\
\hline M & 68 & 2005 & C38.4 & $9053 / 3$ & C38.4 & $9050 / 3$ & 0,9 & $\begin{array}{c}\text { Auxiliares de escritório } \\
\text { e trabalhadores } \\
\text { assemelhados }\end{array}$ \\
\hline$F$ & 69 & 2013 & C48.2 & $9050 / 3$ & C48.2 & $9050 / 3$ & 3,6 & Professor \\
\hline M & 56 & 2001 & C38.4 & $9050 / 3$ & C38.4 & $9050 / 3$ & 79,7 & $\begin{array}{c}\text { Trabalhadores do } \\
\text { comércio e assemelhados }\end{array}$ \\
\hline M & 64 & 2010 & C38.4 & $9050 / 3$ & C34.9 & $9050 / 3$ & 18,9 & $\begin{array}{l}\text { Trabalhadores } \\
\text { metalúrgicos e } \\
\text { siderúrgicos }\end{array}$ \\
\hline M & 59 & 2014 & $\mathrm{C} 38.4$ & $9051 / 3$ & C38.4 & $9051 / 3$ & 6,8 & Trabalhadores de serventia \\
\hline M & 49 & 1998 & C38.4 & $9050 / 3$ & C38.4 & $9050 / 3$ & 6,4 & $\begin{array}{l}\text { Trabalhadores de } \\
\text { produção industrial }\end{array}$ \\
\hline $\mathrm{F}$ & 34 & 1997 & C38.4 & $9051 / 3$ & C38.4 & $9050 / 3$ & 2,2 & Trabalhadores de serventia \\
\hline$F$ & 29 & 2013 & C48.2 & $9050 / 3$ & C48.0 & $9050 / 3$ & 10,2 & $\begin{array}{c}\text { Auxiliares de escritório } \\
\text { e trabalhadores } \\
\text { assemelhados }\end{array}$ \\
\hline M & 63 & 2010 & $\mathrm{C} 38.4$ & $9050 / 3$ & C38.4 & $9050 / 3$ & 13,5 & Técnico em tubulação \\
\hline $\mathbf{F}$ & 74 & 2004 & C38.4 & $9052 / 3$ & C38.4 & $9052 / 3$ & NR & Trabalhadores de serventia \\
\hline M & 61 & 2007 & C38.4 & $9050 / 3$ & C38.4 & $9050 / 3$ & 19,3 & $\begin{array}{l}\text { Trabalhadores } \\
\text { metalúrgicos e } \\
\text { siderúrgicos }\end{array}$ \\
\hline$F$ & 45 & 2007 & C38.4 & $9050 / 3$ & C38.4 & $9050 / 3$ & NR & $\begin{array}{l}\text { Trabalhadores não } \\
\text { classificados }\end{array}$ \\
\hline
\end{tabular}

LEGENDA: P TOPO: código topográfico encontrado no prontuário; P MORFO: código morfológico encontrado no prontuário; RHC TOPO: código topográfico notificado pelo RHC; RHC MORFO: código morfológico notificado pelo RHC NR: não reportado 
A partir da análise das Declarações de Óbito encontradas, foi possível identificar que cinco delas apresentavam como principal causa do óbito diagnósticos diferentes dos encontrados nos prontuários - que servem de base para a notificação junto ao RHC - seja por incompletude de informações ou por utilização de outras nomenclaturas, conforme mostrado no quadro 1.

\section{$\mathbf{N}^{\circ}$ de Casos}

2

1

2
Diagnóstico no prontuário

Mesotelioma pleural

Mesotelioma peritoneal

Mesotelioma

Quadro 1 - Descrição dos diagnósticos encontrados nas Declarações de Óbito e os diagnósticos estabelecidos nos prontuários dos casos de mesotelioma maligno. Curitiba, PR, Brasil, 2017.
Diagnóstico registrado na Declaração de Óbito

Neoplasia maligna da pleura; Câncer de pleura com metástase pulmonar e peritoneal

\section{DISCUSSÃO}

Desde a sua implementação na década de 80 , o sistema RHC possui a responsabilidade de registrar e analisar os casos de câncer atendidos em ambiente hospitalar. Esse registro é realizado de forma específica, levando em consideração a localização topográfica e o comportamento morfológico, bem como o tratamento, recidivas, comportamento metastático e a taxa de mortalidade da doença. O sistema também permite a determinação do perfil sociodemográfico dos casos, o que colabora com o mapeamento das condições de saúde de uma determinada população[14].
Neste estudo, foram encontradas não conformidades nos registros de três dos 16 casos de MM atendidos, nos quais os códigos topográficos utilizados eram incompatíveis com a localização do mesotelioma (C34.9, C48.0 e C49.3). Dessa forma, a conversão final para a CID-10, meio de expressão adotado mundialmente para o acompanhamento do número de casos de todas as doenças, provavelmente não pode ser estabelecido. Tendo isso em vista, a sensibilidade (Se) do RHC para a notificação de casos de MM foi de $81 \%$.

O exame do prontuário não resultou em casos de C38.4 (câncer da pleura). A especificidade (Sp) do registro para diagnóstico 
de câncer da pleura foi de $97 \%$, sendo que quatro dos casos foram codificados erroneamente. Dentre os quatro casos, um se tratava de câncer de pulmão, outro de câncer de mediastino e dois foram relativos à metástase em pleura.

Este resultado corrobora com o estudo realizado no Brasil[15] que mostrou registros de MM dos Estados do Paraná, Santa Catarina e Rio Grande do Sul concluindo que, dos 58 casos registrados com morfologia para MM, 13 casos tinham topografia C34 (pulmão). Entretanto, é possível que a sensibilidade e especificidade do RHC estudado seja diferente de outros RHCs do país, como ocorre com outros sistemas de notificação (SIM, SINASC, SISCOLO), pois, embora sejam adotados há mais de quatro décadas, ainda apresentam variação regional de cobertura e qualidade[16].

Em relação aos códigos morfológicos escolhidos, o código 9050/3 (mesotelioma maligno) foi utilizado genericamente para notificar quatro casos da doença, sendo que em dois casos poderia ter sido utilizado o código 9051/3 (mesotelioma fibroso maligno), em outros dois casos o código 9052/3 (mesotelioma epitelióide maligno) e em outro caso o código 9053/3 (mesote- lioma bifásico maligno). Um resultado análogo foi encontrado na Itália em um estudo que comparou a morfologia entre o Registros de Mesoteliomas e Registros de Câncer de Base Populacional[17].

A escolha da utilização da CID-O3 para a notificação dos casos de câncer em serviços especializados, para repasse de informações diretamente ao INCA, é uma recomendação da IARC. Essa recomendação se deve à possibilidade de detalhamento preciso do câncer, algo desejado pelos oncologistas desde a década de 50 e inviável com os códigos da CID-1[14]. Apesar destes quatro casos de MM terem sido notificados, o objetivo do RHC não foi realizado integralmente.

O uso do RHC é uma experiência recente e exige a adoção de procedimentos de controle de qualidade. As situações consideradas como erro, de acordo com o estabelecido pelo Sis-RHC, requerem reavaliação das informações e validação realizada por consultores da área de oncologia que prestam assessoria aos RHCs. Campos em branco não são aceitos e devem ser preenchidos com o código referente à opção "sem informação"[18]. Neste sentido, uma aliança entre o RHC e os profissionais de saúde que recolhem os dados pode ser 
um caminho a ser percorrido para a geração de informações consistentes, que influenciarão positivamente na tomada de decisões clínicas e administrativas.

No Brasil, foram estimados mais de 596 mil casos novos de câncer para o ano de 2016, correspondendo a segunda causa de morte e com maior incidência na Região Sul[19]. Os avanços nos procedimentos diagnósticos e terapêuticos propiciam maior sobrevida aos pacientes com câncer, mas não necessariamente melhor qualidade de vida pós-tratamento, que só será possível mediante o registro acurado e tomadas de decisões epidemiologicamente fundamentadas.

O Ministério da Saúde do Brasil defende que haverá melhoria na qualidade das informações epidemiológicas levantadas, como consequência direta da melhoria dos registros em prontuários, maior envolvimento e desenvolvimento da equipe de saúde e análise crítica dos dados[20]. Neste contexto, a adoção de estratégias como uso de protocolos, educação permanente, discussão de casos em equipe multidisciplinar e auditoria concorrente podem ser de grande auxílio para o alcance de tal evolução.

Apesar do Brasil ter aprovado leis que proíbem a utilização do amianto, diversos estudos estimam que o número de casos de câncer de pleura e MM aumentará no país em um futuro próximo. Principalmente, em decorrência do período de exposição ao amianto e ao intervalo necessário para o surgimento dos primeiros sinais e sintomas. Ao apontar a eventual subnotificação dessa doença, este estudo contribui socialmente por estimular a realização de treinamentos e capacitações nas instituições notificadoras. Dessa forma, promovendo o fortalecimento de ações que propiciem o diagnóstico precoce e a assistência integral aos indivíduos expostos ao amianto, e potencialmente sujeitos ao adoecimento.

\section{CONCLUSÃO}

Para o Registro Hospitalar de Câncer, todos os casos de MM ocorridos na instituição foram identificados, registrados e notificados. Entretanto, observou-se que em $25 \%$ dos casos, optou-se pela utilização do código morfológico 9050/3, que se refere ao mesotelioma maligno apenas de forma geral. Uma vez que o CID-O3 permite a utilização de outros códigos com o objetivo de detalhar o tipo de MM, torna-se oportuno utilizá-lo de forma a retratar de forma mais especí- 
fica a epidemiologia do MM. Além disso, em situações em que os códigos disponíveis na CID-O3 não foram corretamente atribuídos, a conversão para CID 10 não pôde ser estabelecida, o que provavelmente ocasionou a subnotificação de casos de MM.

Outro ponto de fragilidade na definição do perfil epidemiológico do mesotelioma maligno é o elevado número de informações não preenchidas pelos profissionais de saúde no momento do atendimento aos pacientes. Dessa forma, esse trabalho evidencia a necessidade da conscientização dos profissionais envolvidos na coleta dos dados por meio de ações de educação continuada.

\section{AGRADECIMENTOS}

À Coordenação de Aperfeiçoamento de Pessoal de Nível Superior (CAPES), pela Bolsa Social concedida às autoras, durante o curso de mestrado.

\section{APOIO FINANCEIRO:}

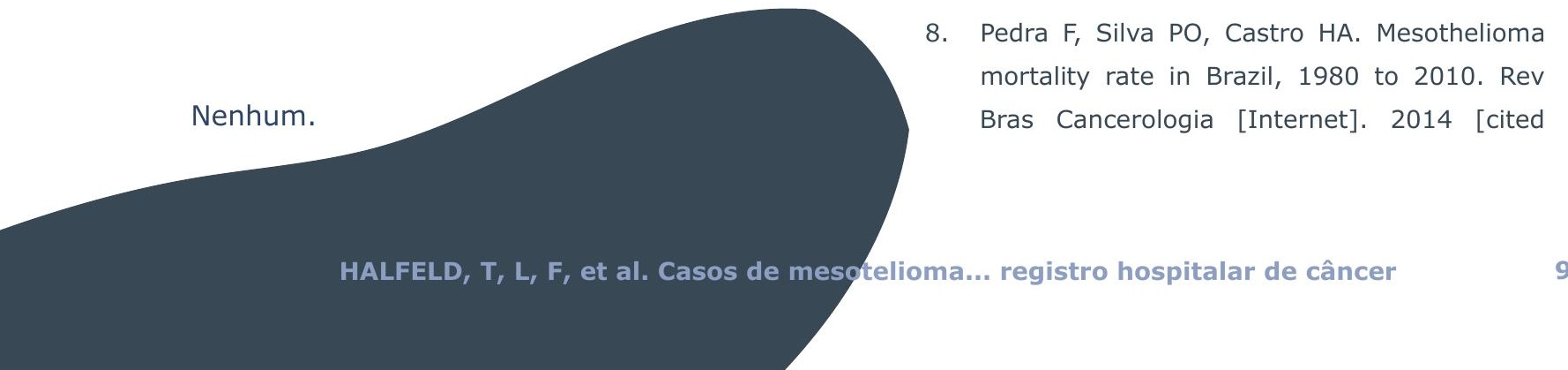

\section{REFERÊNCIAS}

1. Erb CT, Johnson KM, Kim AW. Rare pleural tumors. Clin Chest Med. 2013 Mar; 34(1):11336. doi: $10.1016 /$ j.ccm.2012.12.001.

2. Enzinger FM, Weiss SW. Soft tissue tumors. St. Louis: Mosby; 1995.

3. International Agency for Research on Cancer (IARC). A review of human carcinogens Parte C: Arsenic, Metals, Fibres, and Dusts [Internet]. Lyon/FR: IARC, 2012. v. 100C. [citado 2019 Jun 10]. Available from: https:// publications.iarc.fr/120.

4. Algranti $E$, Saito $C A$, Carneiro AP, Mendonça EM, Bussacos MA. The next mesothelioma wave: mortality trends and forecast to 2030 in Brazil. Cancer Epidemiol. 2015 Oct; 39(5): 687-92. doi: 10.1016/j.canep.2015.08.007.

5. Pawelczyk A, Bozek F. Health risk associated with airbone asbestos. Environ Monit Assess. 2015 Jun; 187(7):428. doi: 10.1007/s10661015-4614-3.

6. Collegium Ramazzini. Asbestos is still with us: repeat call for a universal ban. New Solut. 2010; 20(2):257-66. doi: 10.2190/NS.20.2.j.

7. Ministério da Previdência Social (BR). Empresa de Tecnologia e Informações da Previdência Social (Dataprev). Benefícios por incapacidade concedidos por CID para 2009 [Internet]. Brasília: Ministério da Previdência Social; 2009. [citado 2019 jul 21]. Disponível em: http://www3.dataprev.gov.br/scripts9/ netuno.cgi. 
2019 Mar 10];60(3):199-206. Available from: https://rbc.inca.gov.br/site/arquivos/n_60/ v03/pdf/03-artigo-mesothelioma-mortalityrate-in-brazil-1980-to-2010.pdf

9. Supremo Tribunal Federal (BR). Emenda da Lei Federal 9.055/1995: dispõe sobre a proibição da extração, comercialização e exportação do amianto em território brasileiro. Brasília: Supremo Tribunal Federal; 2017.

10. Mensi C, Matteis SD, Dallari B, Riboldi $L$, Bertazzi PA, Consonni D. Incidence of mesothelioma in Lombardy, Italy: exposure to asbestos, time patterns and future projections. Occup Environ Med. 2016 Sept;73(9):607-13. doi: 10.1136/oemed-2016-103652.

11. Marsili D, Terracini B, Santana VS, RamosBonilla JP, Pasetto R, Mazzeo A, et al. Prevention of asbestos-related disease in countries currently using asbestos. Int J Environ Res Public Health. 2016 May;13(5):494. doi: 10.3390/ijerph13050494.

12. Poblete-Naredo I, Albores A. Molecular biomarkers to assess health risks due to environmental contaminants exposure. Biomedica. 2016 Jun;36(2):309-335. doi: 10.7705/biomedica.v36i3.2998.

13. Ministério da Saúde (BR). Instituto Nacional de Câncer. Câncer no Brasil: dados dos registros de bases populacional [Internet]. Rio de Janeiro: INCA; 2010. v. 4. [citado 2019 jun 15]. Disponível em: https://www.inca. gov.br/sites/ufu.sti.inca.local/files//media/ document//registro_de_base_populacional_ completo.pdf

14. Ministério da Saúde (BR). Instituto Nacional do Câncer. Registros hospitalares de câncer: Planejamento e gestão [Internet]. 2. ed. Rio de Janeiro: INCA; 2010. [citado 2019 jun 15]. Disponível em: http://bvsms.saude.gov.br/ bvs/publicacoes/inca/registros_hospitalares_ cancer.pdf

15. Koller FJ, Sarquis LMM, Mantovani MF, Miranda FMA, Consonni D, Mensi C. Monitoramento do mesotelioma no sul do Brasil: uma realidade ainda a ser estudada. Cogitare Enferm. 2017jan-mar;22(1):01-06. doi: 10.5380/ ce.v22i1.49192

16. Girianelli VR, Thuler LCS, Silva GA. Qualidade do sistema de informação do câncer do colo do útero no estado do Rio de Janeiro. Rev Saúde Pública. 2009 ago;43(4):580-8. doi:10.1590/ S0034-89102009005000043.

17. Nicita C, Buzzoni C, Chellini E, Ferrretti S, Marinaccio A, Mensi $C$, et al. A comparative analysis between regional mesothelioma registries and cancer registries: results of the ReNaM-AIRTUM project. Epidemiol Prev [Internet]. 2014 [citado 2019 Mar 10];38(34):191-9. Disponível em: https://www.ncbi. nIm.nih.gov/pubmed/25115471

18. Pinto IV, Ramos DN, Costa MCE, Ferreira CBT, Rebelo MS. Completude e consistência dos dados dos registros hospitalares de câncer no Brasil. Cad Saúde Coletiva [Internet]. 2012 [citado 2019 jun 15];20(1):113-20. Disponível em: http://www.cadernos.iesc. ufrj.br/cadernos/images/csc/2012_1/artigos/ CSC_v20n1_113-120.pdf

19. Ministério da Saúde (BR). Instituto Nacional do Câncer. Estimativa 2016: incidência de câncer no Brasil [Internet]. Rio de Janeiro: INCA; 2015. [citado 2019 jun 15]. Disponível em: http://santacasadermatoazulay.com. br/wp-content/uploads/2017/06/estimativa2016-v11.pdf

20. Ministério da Saúde (BR). Departamento de Ciência e Tecnologia; Secretaria de Ciência e Tecnologia e Insumos Estratégicos. 
Integração de informações dos registros de câncer brasileiros. Rev Saúde Pública. 2007 out;41(5):865-8. doi: http://dx.doi. org/10.1590/S0034-89102007000500024. 